\title{
Escala de Avaliação de Comportamento Sexual de Risco para Adultos: tradução e adaptação transcultural para o português brasileiro
}

\author{
Sexual Risk Behavior Assessment Schedule for Adults: translation and cross- \\ cultural adaptation into Brazilian Portuguese
}

\author{
Diana de Souza Pinto ${ }^{1}$, Carlos Linhares Veloso Filho ${ }^{2}$, Milton L. Wainberg ${ }^{3}$, Paulo Eduardo Luís \\ de Mattos ${ }^{4}$, Heino F. L. Meyer-Bahlburg ${ }^{5}$
}

${ }^{1}$ Doutora. Universidade Federal do Rio de Janeiro (UFRJ), Rio de Janeiro, RJ. ${ }^{2}$ Mestre, Saúde Coletiva, UFRJ. ${ }^{3}$ Associate Clinical Professor of Psychiatry, Columbia University, NY, EUA. ${ }^{4}$ Pós-Doutorado, Psiquiatria, UFRJ. ${ }^{5}$ Doutor, Psicologia Clínica. Associate director, HIV Center for Clinical and Behavioral Studies, New York State Psychiatric Institute, Columbia University, New York, NY, EUA.

\section{Resumo}

Introdução: A investigação sistemática e detalhada dos comportamentos sexuais de risco constitui um dos principais alicerces para o desenvolvimento de pesquisas que visam à criação de intervenções eficazes para a prevenção do HIV.

Objetivo: Descrever e discutir as etapas do processo de adaptação lingüístico-cultural das versões masculina e feminina da Escala de Avaliação de Comportamento Sexual de Risco para pacientes psiquiátricos para o português brasileiro pelo Projeto Interdisciplinar em Sexualidade, Saúde Mental e AIDS

Método: Treinamento dos investigadores brasileiros no instrumento original, comparação da tradução e da retrotradução, seguida de adaptação lingüístico-cultural à luz da fase formativa do projeto, resultando em uma versão utilizada no treinamento dos entrevistadores brasileiros em entrevistas com pacientes psiquiátricos. Verificação lingüística, confiabilidade qualitativa do instrumento e confiabilidade entre entrevistadores constituíram as etapas complementares para a versão final do questionário.

Resultados: Exclusão e adição de seções e itens da escala, tanto nos aspectos conceituais quanto estruturais, foram realizadas, bem como a incorporação de achados resultantes da fase formativa do piloto da pesquisa e mudanças lingüísticas relativas ao registro empregado.

Descritores: Questionários, comportamento de risco, comparação transcultural, prevenção de doenças transmissíveis, estudos de intervenção.

\begin{abstract}
Introduction: A systematic and detailed investigation of sexual risk behaviors is one of the main foundations in the development of research projects aimed at designing effective interventions for HIV prevention.

Objective: This paper presents and discusses the stages of the translation and cross-cultural adaptation of male and female versions of the Sexual Risk Behavior Assessment Schedule for psychiatric patients into Brazilian Portuguese, carried out by the Interdisciplinary Project in Sexuality, Mental Health and AIDS.

Methods: Training of Brazilian investigators in the original questionnaire, comparison between translation and back-translation, followed by linguistic and cultural adaptation in the light of the project formative phase were performed, resulting in a version used to train Brazilian interviewers in interviewing psychiatric patients. Language verification, qualitative reliability and interrater reliability were used to complement the final version of the instrument.

Results: Elimination and addition of instrument sections and items covering both conceptual and structural aspects were carried out. Incorporation of formative findings from the pilot study and linguistic changes in terms of register were also performed.

Keywords: Questionnaires, risk behavior, cross-cultural comparison, communicable disease prevention, intervention studies.
\end{abstract}




\section{Introdução}

Os estudos destinados à criação de intervenções para a prevenção do HIV têm se deparado com vários desafios nas últimas décadas. As formas de propagação do vírus, tais como o uso de drogas injetáveis e o engajamento em relações sexuais sem uso de preservativos, são, com freqüência, associadas a comportamentos e atitudes discriminadas social e culturalmente $^{1-3}$, bem como a concepções estereotipadas dos outrora chamados grupos de risco ${ }^{4}$. O conhecimento detalhado e sistemático dos comportamentos sexuais de risco é um dos aspectos fundamentais para o desenvolvimento de pesquisas que visam à criação de programas eficazes para a prevenção do HIV. Tal situação torna-se premente quando tratamos de populações extremamente vulneráveis ao vírus, como no caso de portadores de transtorno mental grave (PTMG), cujas taxas são 3 a 5 vezes maiores do que as da população em geral ${ }^{5}$. Compreendemos PTMG como indivíduos com diagnósticos de esquizofrenia, transtorno esquizoafetivo, transtorno esquizofreniforme, transtorno bipolar, transtorno depressivo maior com sintomas psicóticos e psicose não-especificada. No Brasil, especificamente em Belo Horizonte, Almeida ${ }^{6}$, em seu estudo inédito de soroprevalência com usuários dos serviços de saúde mental, encontrou taxas cinco vezes maiores do que para a população em geral.

O Projeto Interdisciplinar em Sexualidade, Saúde Mental e AIDS (PRISSMA) é um estudo colaborativo - Universidade de Columbia (EUA), Instituto de Psiquiatria da Universidade Federal do Rio de Janeiro (IPUB/UFRJ) e Associação Brasileira Interdisciplinar de AIDS (ABIA) - financiado pelo National Institute of Mental Health (NIMH) (protocolo $\mathrm{n}^{\circ}$ R01MH65163), que avaliou a viabilidade de se criar e aplicar uma nova intervenção para prevenção da infecção pelo HIV em homens e mulheres usuários de saúde mental.

Intervenções americanas eficazes para a prevenção do HIV, já testadas nos $\mathrm{EUA}^{7-12}$, foram adaptadas através de um modelo de pesquisa participatória ${ }^{13}$, com base nas características e necessidades culturais locais, investigadas através de métodos etnográficos e de adaptação cultural ${ }^{14}$. Esse processo de adaptação cultural obedeceu às etapas necessárias observadas pelos pesquisadores, após extensa revisão da literatura em adaptação de intervenções ${ }^{15-18}$.

Face à ausência de instrumentos padrão brasileiros para a avaliação de comportamentos sexuais de risco nessa população, o PRISSMA realizou a adaptação transcultural das versões masculina e feminina da Escala de Avaliação de Comportamento Sexual de
Risco (SERBAS) para adultos com PTMG, instrumento de coleta de informações sobre tais comportamentos especificamente nos três últimos meses $^{19}$.

O objetivo do presente artigo é descrever as etapas do processo de adaptação lingüístico-cultural desse instrumento que resultaram nas versões masculina e feminina finais adotada pelo PRISSMA em sua primeira fase ${ }^{14,20,21}$. Essas versões também serão utilizadas na etapa posterior, o estudo clínico randomizado, submetido ao NIMH.

\section{Descrição do instrumento}

A SERBAS, nas versões masculina e feminina, é uma entrevista semi-estruturada, tendo, em sua maioria, perguntas fechadas. Ela foi originalmente desenvolvida e validada nos EUA, com o objetivo de coletar informações detalhadas das práticas sexuais e do uso de álcool e drogas relacionados a elas, tendo sido empregada em pesquisas com diferentes populações, incluindo PTMG de ambos os sexos ${ }^{11,19,22-}$ ${ }^{24}$. A SERBAS avalia comportamentos sexuais de risco nos últimos 3 meses, e a versão original americana foi o resultado de uma compilação de outras versões desenvolvidas para diferentes projetos de pesquisa, onde foram consideradas características das populações investigadas (idade, gênero, orientação sexual, etnia), o foco dos estudos (definido pelos resultados e pelas variáveis dependentes), detalhes de comportamento sexual investigado e o tempo disponível para a aplicação do instrumento. A SERBAS revelou-se um instrumento flexível, na medida em que permite adaptações necessárias às várias questões próprias das pesquisas.

A SERBAS original, utilizada com PTMG, contém 60 perguntas, com quatro tabelas, para facilitar o registro das várias informações pelo entrevistador. $\mathrm{Na}$ introdução, há instruções para o entrevistador sobre como explicar o tema da entrevista de modo a deixar o participante à vontade; segue-se a seção de "terminologia", onde termos das partes do corpo e de práticas sexuais (que serão utilizados ao longo da entrevista) são explicados. Para cada um dos termos (por exemplo: vagina, pênis, etc.), é pedido ao entrevistado que apresente os seus correlatos de preferência, que serão, então, empregados ao longo de toda a entrevista. A seguir, há uma seção de perguntas sobre parceiros do sexo oposto que inclui a definição do tipo de parceiro (se casual ou fixo), conhecimentos sobre a sorologia do(a) parceiro(a) e práticas sexuais (sexo vaginal, oral e anal) com cada um daqueles e eventuais proteções utilizadas, bem como as trocas sexuais (sexo por dinheiro, drogas ou abrigo). A seção seguinte, de estrutura semelhante, avalia o 
conhecimento das práticas sexuais com parceiros do mesmo sexo, quando houver. Perguntas sobre métodos de proteção e prevenção integram a penúltima seção, seguida de um encerramento e de uma avaliação do entrevistador acerca da qualidade da entrevista. A duração média da aplicação das duas versões dependeu do número de parceiros e de atividades sexuais que os participantes relataram. A avaliação detalhada ${ }^{22} \mathrm{da}$ SERBAS acerca de comportamentos sexuais, uso de preservativo, tipo de parceiros, trocas sexuais e uso de substâncias associadas ao risco possibilita uma gama de variáveis resultantes para posterior análise.

A confiabilidade das entrevistas de seguimento de 3 meses para avaliação de variáveis-chave para comportamentos sexuais de risco foi considerada entre aceitável e excelente em estudos com populações marginais, a exemplo dos PTMG sem-teto ${ }^{25}$, pacientes psiquiátricos internados e ambulatoriais ${ }^{19}$ e adolescentes urbanos homossexuais ${ }^{26,27}$.

\section{Método}

1) Adaptação transcultural

A adaptação lingüístico-cultural da SERBAS, nas versões masculina e feminina, para o português do Brasil, desenvolvida pela equipe do PRISSMA, aprovada pelos autores, seguiu as seguintes etapas metodológicas, apresentadas na Figura 1 e descritas a seguir:

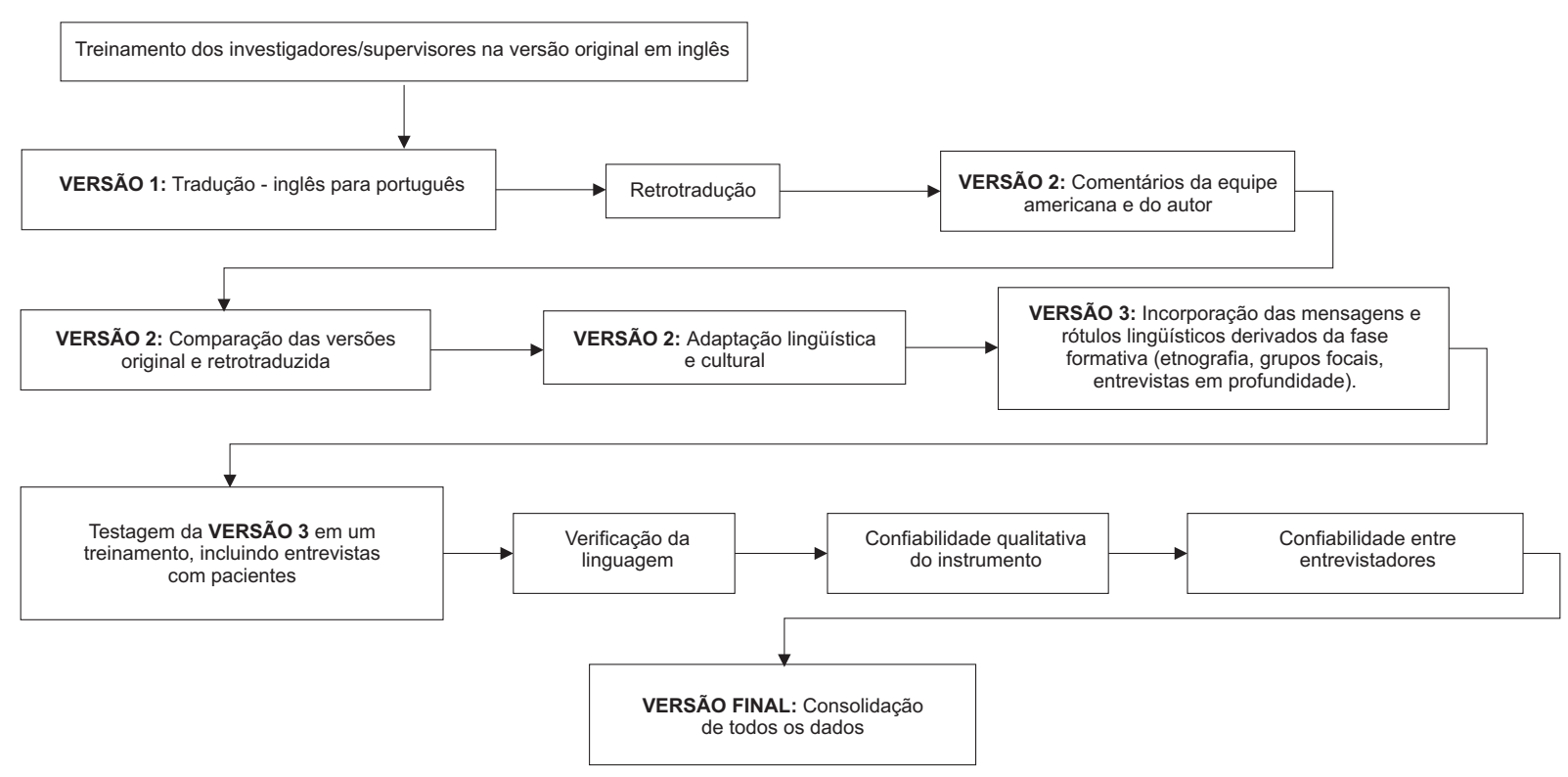

a) Treinamento de membros brasileiros da equipe de pesquisa nos EUA pelos autores do instrumento. Foram realizadas entrevistas com PTMG ambulatoriais sexualmente ativos em duas instituições psiquiátricas diferentes na cidade de Nova Iorque, sob supervisão e discussão acerca de questões de adaptação, com vistas a futuras comparações com outros projetos de pesquisa nos quais a SERBAS foi utilizada.

b) Tradução para o português brasileiro e posterior retrotradução para o inglês por duas equipes distintas de tradutores experientes bilíngües para permitir controle de qualidade; as versões original e retrotraduzida foram comparadas por dois pesquisadores bilíngües do projeto e autores deste artigo, com experiência em pesquisas em Saúde Mental, um antropólogo, que já havia atuado como entrevistador do instrumento original, na versão masculina ${ }^{11,23}$, e uma lingüista, para avaliar discrepâncias de conteúdo, forma e de apresentação/ formatação. As discrepâncias encontradas nas duas versões em inglês foram contrastadas para garantir a correspondência de tradução de sentido e analisadas por dois pesquisadores bilíngües - a lingüista que participara da etapa anterior e um psiquiatra com experiência em clínica e pesquisa. O passo seguinte foi garantir a adequação do instrumento ao contexto das populações locais.

c) Adaptação lingüístico-cultural com base nos resultados da fase formativa do PRISSMA ${ }^{14}$, na qual foram utilizados métodos etnográficos (observações etnográficas, grupos focais e entrevistas com informantes-chave) que geraram uma ampla 
compreensão das crenças, normas e atitudes relativas a sexualidade, identidade sexual e comportamentos sexuais (de risco) ${ }^{28}$. A comparação transcendeu o sentido literal de palavras, expressões e estruturas, na medida em que incorporou aspectos socioculturais envolvidos em qualquer situação de língua em uso ${ }^{29}$. Os termos apresentados na seção inicial de terminologia, empregada para descrever as partes do corpo, práticas e atividades sexuais, receberam especial atenção, face ao possível impacto sobre a população-alvo e à importância dessa seção para todo o desenvolvimento da entrevista. As adaptações de registro lingüístico e estilo foram consideradas fundamentais, já que as perguntas deveriam ser claras e rapidamente compreendidas pelos entrevistados. As discrepâncias foram registradas, e as seções em português foram então revisadas por dois pesquisadores bilíngües da equipe brasileira para garantir acuidade. Mensagens e rótulos lingüísticos derivados dos grupos focais e das entrevistas foram incorporados posteriormente pelos mesmos pesquisadores bilíngües da equipe brasileira responsáveis pela etapa anterior.

d) Treinamento ${ }^{30}$ da segunda versão, num total de 30 horas com os dois pesquisadores assistentes da pesquisa, graduados em psicologia, responsáveis por realizar as entrevistas da SERBAS com pacientes da clínica-satélite, selecionados aleatoriamente, coordenado pelo pesquisador coordenador da equipe americana, que já tinha atuado como entrevistador do questionário em estudos anteriores ${ }^{11}$. Esse treinamento objetivou familiarizar os entrevistadores com a correta seqüência das seções do instrumento, destacando as maneiras mais adequadas de fazer as perguntas para deixar os entrevistados mais à vontade na investigação de aspectos relativos a identidade, práticas e atividades sexuais.

e) Essa segunda versão foi testada entre os dois pesquisadores assistentes da pesquisa - dois psicólogos de ambos os sexos - sob a supervisão do pesquisador psiquiatra. Este ajudou na consolidação dessa versão adaptada, com vistas à avaliação do conforto dos entrevistadores para abordar os temas do instrumento. Todas as entrevistas com os 10 pacientes (cinco mulheres e cinco homens) do hospital-dia da clínica-satélite foram gravadas em vídeo, com a câmera enquadrando apenas o entrevistador, para manter a confidencialidade dos dados, de acordo com o termo de consentimento informado, lido e assinado pelos entrevistados. As entrevistas também eram monitoradas por outro entrevistador, que tinha o seu próprio instrumento para registro das informações, posteriormente comparado com o registro do entrevistador que conduziu a entrevista. Os focos dessa etapa eram examinar e analisar o conforto dos entrevistadores na situação real de entrevista com pacientes psiquiátricos e observar a qualidade das informações obtidas. O registro em áudio dessas entrevistas e as anotações eram discutidas em sessões de supervisão em grupo, realizadas posteriormente. A constante troca de experiências com a entrevista, baseada nos feedbacks dos entrevistadores, permitiulhes padronizar a aplicação do instrumento, particularmente no item relativo à investigação de um fato marcante ocorrido com o entrevistado 3 meses antes da coleta de dados, período a ser usado como ponto de referência dos comportamentos sexuais de risco ("Você consegue se lembrar de qualquer coisa que aconteceu na sua vida há mais ou menos 3 meses que faça este período ficar bem marcante para você?").

f) Foi realizada uma verificação lingüística (debriefing), com o objetivo de analisar em que medida os entrevistados compreenderam o conteúdo de cada uma das perguntas. Para essa etapa, foram entrevistados seis pacientes, de ambos os sexos, do hospital-dia da clínica-satélite. Logo após cada resposta do entrevistado para cada uma das perguntas do instrumento, o entrevistador perguntava: "O que acabei de perguntar a você?". Esse breve espaço de tempo entre a pergunta do instrumento e a verificação de sua compreensão foi considerada fundamental face às limitações de memória que freqüentemente acompanham alguns dos transtornos psiquiátricos dessa população. Discrepâncias sobre a compreensão da pergunta eram então registradas por escrito pelo entrevistador e pelo observador/pesquisador assistente da entrevista. As anotações e os registros em áudio subsidiaram as reuniões de supervisão em grupo nas quais os mal-entendidos foram discutidos.

g) Os resultados da etapa anterior foram incorporados em uma terceira e final versão, submetida à avaliação da confiabilidade através do procedimento de teste/reteste. Nove pacientes, de ambos os sexos, foram entrevistados duas vezes no período de até 1 semana para verificar a replicação e qualidade das respostas por diferentes entrevistadores. Os resultados de ambas as entrevistas foram comparados. A versão brasileira da SERBAS (SERBAS-B) mostrou excelente confiabilidade teste/reteste para os últimos 3 meses com relação à atividade sexual $($ kappa $=1,0)$ e razoável a boa concordância na freqüência de sexo anal e vaginal, incluindo ocasiões de sexo desprotegido (correlação intraclasse $=0,5-1,0$ ). Esses resultados são consistentes com estudos prévios de confiabilidade em PTMG nos EUA, que examinaram comportamentos sexuais durante os 6 meses anteriores ${ }^{19,25}$.

A partir da quinta entrevista, ainda durante essa fase, procedeu-se à avaliação da confiabilidade entre entrevistadores (interrater reliability). Esse procedimento foi realizado mensalmente ao longo de 
todo o estudo. A versão final adaptada para o português do Brasil não foi retrotraduzida, e foram utilizados registros em áudio com o objetivo de averiguar se todos os entrevistadores obtinham as mesmas informações dos mesmos entrevistados em todas as seções do instrumento. Todos os entrevistadores deveriam assinalar individualmente as respostas com base no áudio da entrevista e depois compará-las em grupo. Nessa etapa, observou-se pequenas discrepâncias, a exemplo da caracterização dos tipos de parceiro (fixo, casual ou troca). As discrepâncias foram então discutidas pela equipe; no exemplo citado, reiterou-se que a definição do tipo de parceiro cabia sempre ao entrevistado. A escolha entre uma das opções - fixo, casual ou troca - para cada um dos parceiros é de natureza subjetiva. Assim, um(a) profissional do sexo pode ser caracterizado(a) sob qualquer um dos três tipos.

\section{Resultados e discussão}

Dada a extensão da SERBAS, agrupamos as modificações feitas no instrumento nas três categorias abaixo:

a) Aspectos conceituais/estruturais: eliminação/ adição de itens, temas e perguntas. Seções ou perguntas que não endereçavam especificamente comportamento sexual de risco foram eliminadas, como, por exemplo: "métodos de prevenção e proteção", que investigava os diferentes métodos contraceptivos da entrevistada ou da parceira do entrevistado e questões referentes à "inserção vaginal/anal de objetos". Outras partes também foram retiradas por não abordarem objetivamente práticas de risco, como, por exemplo, as perguntas sobre o comportamento do(a) parceiro(a) - se tinha sintomas de doenças sexualmente transmissíveis (DST) e se já fora preso(a). Outras questões referiam-se a circunstâncias que apenas tangencialmente poderiam estar relacionadas ao alvo do instrumento, a exemplo da seguinte questão no instrumento para mulheres: "Nos últimos 3 meses, você sentiu alguma dor na vagina ou no abdômen enquanto fazia sexo vaginal?".

Na seção de terminologia, "clitóris" era um termo empregado em perguntas ao longo do instrumento, porém não estava listado/definido originalmente, tendo sido adicionado na versão brasileira. A distinção existente no instrumento original entre número de "vezes" e número de "ocasiões" em que a pessoa fez sexo foi também excluída por ser mal compreendida pelos entrevistados. Nessa distinção, "ocasião" é entendida como número de encontros de natureza sexual, diferentemente de "vezes", compreendida como número de penetrações/contatos sexuais ocorridos em cada uma das ocasiões. b) Aspectos oriundos dos achados da fase formativa da pesquisa e/ou estudos prévios. O termo travesti/transexual foi adicionado, e as eventuais práticas sexuais com essa população foram computadas na tabela final de acordo com a classificação de gênero atribuída pelo(a) entrevistado(a) ao travesti/transexual. A "praia" foi acrescentada como opção de local de encontro, tendo em vista o estudo realizar-se em uma região litorânea. $\mathrm{O}$ instrumento original também pedia informações específicas sobre práticas sexuais dos últimos 3 meses com três parceiros(as). Na versão adaptada, o entrevistador é instruído a coletar informações de até 10 parceiros(as), número que resultou também das várias entrevistas realizadas durante a adaptação do instrumento. Na questão sobre uso de preservativos para qualquer tipo de prática sexual (sexo vaginal, anal e oral), caso o entrevistado respondesse que não utilizou, o entrevistador deveria perguntar-lhe a razão do não-uso, registrando exatamente as palavras utilizadas pelo entrevistado em sua resposta. Logo após a seção de terminologia, o instrumento original perguntava ao(à) entrevistado(a) sua identidade sexual, "como você se vê: como heterossexual, gay, lésbica, bissexual ou outra opção?" (que deveria ser então especificada). Contudo, alguns estudos sobre gênero e sexualidade ${ }^{31}$ sugerem que a categoria identidade sexual, em parte da população brasileira, é construída segundo parâmetros de atividade/ passividade sexual, no caso do gênero masculino. $\mathrm{Na}$ fase formativa, freqüentes relatos de práticas sexuais de entrevistados de ambos os gêneros com parceiro(a)s do mesmo sexo, particularmente nos períodos de internação em instituições psiquiátricas, não implicaram em mudanças de autopercepção da identidade sexual. Assim, a pergunta foi eliminada da versão adaptada. $\mathrm{O}$ uso e o valor de troca do cigarro em instituições psiquiátricas, já apontados em vários estudos de cunho etnográfico ${ }^{32}$, evidentes nas várias etapas da fase formativa do estudo do PRISSMA, foram integrados à pergunta sobre trocas sexuais ("Com quantos parceiros(as) você fez sexo em troca de cigarros?").

c) Mudanças lingüísticas: além da padronização das instruções para os entrevistados, nas versões masculina e feminina, que originalmente foram criadas em momentos e com propósitos distintos, houve a preocupação com a objetividade e clareza das perguntas, optando-se sempre por formas comuns no registro oral informal do português do Brasil. $\mathrm{Na}$ seção de parceiras mulheres, no instrumento feminino, por exemplo, pergunta-se: "Outras vezes, elas (as mulheres) transam com mulheres em troca de dinheiro, de um lugar para ficar ou para ajudar nas despesas". 


\section{Conclusão}

A adaptação transcultural de instrumentos é reconhecidamente um processo múltiplo e complexo, que exige cuidados metodológicos de diferentes naturezas. A necessidade de adequação ao contexto cultural alvo é apontada por vários autores como uma etapa fundamenta ${ }^{33,34}$. O emprego de metodologias de base etnográfica, a exemplo dos grupos focais, sugerido pela Organização Mundial da Saúde para adaptação de instrumentos de avaliação de qualidade de vida $^{35}$, mostrou-se fundamental no processo de adaptação da SERBAS. Como apontado por Pechansky et al. ${ }^{1}$, em estudo de adaptação e validação da versão brasileira de um questionário sobre comportamento de risco para a AIDS para usuários de drogas, o conhecimento de fatores culturais da realidade local da população-alvo contribuiu consideravelmente para a adaptação aqui descrita, na medida em que informou quais aspectos relativos aos comportamentos sexuais de risco e fatores associados da população psiquiátrica deveriam ser modificados e/ ou incorporados.

Apesar de a versão final adaptada para o português do Brasil não ter sido retrotraduzida, segundo orientações preconizadas na área ${ }^{33,35}$, acreditamos que a utilização da versão brasileira desse instrumento permitirá uma compreensão pormenorizada dos comportamentos de risco e fatores associados nessa população. A SERBAS versão português possibilitounos comparar os termos usados para os comportamentos de risco de pacientes brasileiros(as) e de outros países, a exemplo dos EUA ${ }^{19}$. Dessa forma, a adaptação transcultural da SERBAS adulta constitui uma etapa de fundamental importância para o avanço de pesquisas de criação de intervenções de prevenção para o HIV/DST nessa população tão vulnerável.

\section{Referências}

1. Pechansky F, Hirakata V, Metzger D. Adaptation and validation of a questionnaire about risk behaviors for AIDS among drug users. Rev Bras Psiquiatr. 2002;24(3):130-6.

2. Simões AM, Bastos FI. Audio Computer-Assisted Interview: a new technology in the assessment of sexually transmitted diseases, HIV, and drug use. Cad Saude Publica. 2004;20(5):1169-81.

3. Riley ED, Chaisson RE, Robnett TJ, Vertefeuille J, Strathdee SA, Vlahov D. Use of Audio Computer-assisted Self-Interviews to Assess Tuberculosis-related Risk Behaviors. Am J Respir Crit Care Med. 2001;164(1):82-5.

4. Grangeiro A, Ferraz D, Barbosa R, Barreira D, Veras MASM, Villela W, et al. UNGASS-HIV/Aids: balanço da resposta brasileira, 2001-2005. Rev Saude Publica. 2006;40(Supl):5-8.

5. McKinnon K, Cournos F, Herman R, Le Melle S. HIV and people with serious and persistent mental illness. In: Citron K, Brouillette MJ, Beckett A, editors. HIV \& psychiatry: a training and resource manual. Cambridge: Cambridge University; 2005. p. 138-52.

6. Almeida RC, Pedroso ERP. Vulnerabilidade e exposição a marcadores sorológicos dos vírus da imunodeficiência humana, hepatites B e C, vírus linfotrópico de células T humanas e sífilis em pacientes psiquiátricos internados em hospital público. Rev Med Minas Gerais. 2004;14(4):244-50.

7. Carey MP, Carey KB, Maisto SA, Gordon CM, Schroder KE, Vanable PA. Reducing HIV-risk behavior among adults receiving outpatient psychiatric treatment: Results from a randomized controlled trial. J Consult Clin Psychol. 2004;72(2):252-68.

8. Kalichman S, Sikkema K, Kelly J, Bulto M. Use of a brief behavioral skills intervention to prevent HIV infection among chronic mentally ill adults. Psychiatr Serv. 1995;46(3):275-80.

9. Kelly JA, McAuliffe TL, Sikkema KJ, Murphy DA, Somlai AM, Mulry G, et al. Reduction in risk behavior among adults with severe mental illness who learned to advocate for HIV prevention. Psychiatr Serv. 1997;48(10):1283-8

10. Otto-Salaj L, Kelly J, Stevenson L, Hoffmann R, Kalichman, S. Outcomes of a randomized small group HIV prevention intervention trial for people with serious mental illness. Community Ment Health J. 2001;37(2):123-44.

11. Susser E, Valencia E, Berkman A, Sohler N, Conover S, Torres J, et al. Human immunodeficiency virus sexual risk reduction in homeless men with mental illness. Arch Gen Psychiatry. 1998;55(3):266-72.

12. Weinhardt LS, Carey MP, Carey KB, Verdecias RN. Increasing assertiveness skills to reduce HIV risk among women living with a severe and persistent mental illness. J Consult Clin Psychol. 1998;66:680-4.

13. Cornwall A, Jewkes R. What is participatory research? Soc Sci Med. 1995;41(12):1668-76.

14. Wainberg ML, Gonzalez AM, McKinnon K, Elkington K, Pinto D, Mann CG, Mattos PE. Targeted ethnography as a critical step to inform cultural adaptations of HIV prevention interventions for adults with severe mental illness. Soc Sci Med. 2007;65(2):296308 .

15. Bauman LJ, Stein RE, Ireys HT. Reinventing fidelity: The transfer of social technology among settings. Am J Community Psychol. 1991;19(4):619-39.

16. Kelly JA, Heckman TG, Stevenson LY, Williams PN, Ertl T, Hays $\mathrm{RB}$, et al. Transfer of research-based HIV prevention interventions to community service providers: Fidelity and adaptation. AIDS Educ Prev. 2000;12(5 Suppl):87-98.

17. Kumpfer KL, Alvarado R, Smith P, Bellamy N. Cultural sensitivity and adaptation in family-based prevention interventions. Prev Sci. 2002;3(3):241-6.

18. Rogler LH. Methodological sources of cultural insensitivity in mental health research. Am Psychol. 1999;54(6):424-33.

19. McKinnon K, Cournos F, Meyer-Bahlburg HFL, Guido JR, Caraballo LR, Margoshes ES, et al. Reliability of sexual risk behavior interviews with psychiatric patients. Am J Pshychiatry. 1993;150(6):972-4.

20. Pinto DS, Mann CG, Wainberg M, Mattos P, Oliveira SB. Sexualidade e vulnerabilidade para o HIV em saúde mental: um estudo de base etnográfica de instituições psiquiátricas. Cad Saude Publica. 2007;23(9):2224-33.

21. Wainberg ML, McKinnon K, Mattos PE, Pinto D, Mann CG, Santos de Oliveira CS, et al. A model for adapting evidence-based behavioral interventions to a new culture: HIV prevention for psychiatric patients in Rio de Janeiro, Brazil. In: AIDS Behav., 2007. Disponível na URL: http://www.ncbi.nlm.nih.gov/sites/ e $\mathrm{ntrez}$ ? c m d $=\mathrm{R}$ e tri e v e $\& \mathrm{~d} b=\mathrm{p} \mathrm{u} \mathrm{b} \mathrm{m} \mathrm{e} \mathrm{d} \mathrm{\&} \mathrm{d} \mathrm{o} \mathrm{p} \mathrm{t}=$ Abstract\&list_uids $=17216334$

22. Meyer-Bahlburg H, Ehrhardt A, Exner TM, Gruen RS. Sexual risk behavior assessment schedule-adult-armory interview. New York: Psychiatric Institute and Columbia University; 1991.

23. Susser E, Valencia E, Miller M, Tsai WY, Meyer-Bahlburg HF, Conover S. Sexual behaviors of homeless mentally ill men at risk for HIV. Am J Psychiatry; 1995;152(4):583-7.

24. Collins PY, Geller PA, Miller S, Toro P, Susser ES. Ourselves, our bodies, our realities: an HIV prevention intervention for women with severe mental illness. J Urban Health. 2001;78(1):162-75

25. Sohler N, Colson P, Meyer-Bahlburg HF, Susser E. Reliability of self-reports about sexual risk behavior for HIV among homeless 
men with severe mental illness. Psychiatr Serv. 2000;51(6):814-6.

26. Hearn KD, O'Sullivan LF, Dudley CD. Assessing reliability of urban adolescent girls reports of sexual and romantic behavior. Arch Sex Behav. 2003;32(6):513-21

27. Schrimshaw EW, Rosario M, Meyer-Bahlburg HF, Scharf-Matlick AA. Test-retest reliability of self-reported sexual behavior, sexual orientation, and psychosexual milestones among gay, lesbian, and bisexual youths. Arch Sex Behav. 2006;35(2):225-34.

28. Parker R, Herdt G, Carballo M. Sexual culture, HIV transmission, and AIDS research. J Sex Res. 1991;28:77-98.

29. Gumperz JJ. Discourse strategies. Cambridge: Cambridge University. 1982.

30. Dugan TM, Meyer-Bahlburg HFL. A training program for sex research interviewers. In: di Mauro D, Herdt G, Parker R (eds.), Handbook of sexuality research training initiatives. New York, NY:
Social Science Research Council; 2003. p. 80-92. Disponível em URL: http://nsrc.sfsu.edu

31. Parker R. Corpos, prazeres e paixões: cultura sexual no Brasil contemporâneo. São Paulo: Best Seller; 1991.

32. Goffman E. Manicômios, prisões e conventos. São Paulo: Perspectiva; 1961

33. Caron J. Un guide de validation transculturelle dês instruments de mesure em santé mentale. 1998. Disponível em URL: http:// www.rsmq.qc.ca/fr/instruments/guide.htm.Jorge

34. Meyer E, Carvalhal A, Pechansky F. Adaptation for Brazilian Portuguese of a scale to measure willingness to wear condoms. Rev Bras Psiquiatr. 2003;25(4):224-7.

35. Fleck MPA, Leal OF, Louzada S, Xavier M, Chachamovich E, Vieira G, et al. Development of the portuguese version of the OMS evaluation instrument of quality of life. Rev Bras Psiquiatr. 1999;21(1):19-28. 